\title{
NUMERICALLY GORENSTEIN SURFACE SINGULARITIES ARE HOMEOMORPHIC TO GORENSTEIN ONES
}

\author{
PATRICK POPESCU-PAMPU
}

\begin{abstract}
Consider a normal complex analytic surface singularity. It is called Gorenstein if the canonical line bundle is holomorphically trivial in some punctured neighborhood of the singular point and numerically Gorenstein if this line bundle is topologically trivial. The second notion depends only on the topological type of the singularity. Laufer proved in 1977 that, given a numerically Gorenstein topological type of singularity, every analytical realization of it is Gorenstein if and only if one has either a Kleinian or a minimally elliptic topological type. The question to know if any numerically Gorenstein topology was realizable by some Gorenstein singularity was left open. We prove that this is indeed the case. Our method is to plumb holomorphically meromorphic 2-forms obtained by adequate pull-backs of the natural holomorphic symplectic forms on the total spaces of the canonical line bundles of complex curves. More generally, we show that any normal surface singularity is homeomorphic to a $\mathbb{Q}$-Gorenstein singularity whose index is equal to the smallest common denominator of the coefficients of the canonical cycle of the starting singularity.
\end{abstract}

\section{INTRODUCTION}

Let $(X, 0)$ be a normal complex analytic surface singularity. It is called Gorenstein if the canonical line bundle of some representative of it is holomorphically trivial outside 0 , and numerically Gorenstein if the same line bundle is topologically trivial outside 0. For example, hypersurfaces and complete intersections with isolated singularities are normal and Gorenstein.

One has fundamental systems of neighborhoods of $0 \in X$ which are homeomorphic to cones over an oriented 3-manifold, called the boundary or the link of $(X, 0)$. By a theorem of Neumann [13, the knowledge of the boundary up to oriented homeomorphisms is equivalent to the knowledge of the dual graph of the exceptional divisor of any good resolution, weighted by the genera and the self-intersections of its irreducible components. We say that two singularities are homeomorphic if and only if they have boundaries which are oriented homeomorphic. By Neumann's theorem, this means that they have good resolutions with isomorphic weighted dual graphs.

The canonical cycle of a resolution of the surface singularity is by definition the unique cycle supported by the exceptional divisor $E$, which has the same intersections with the irreducible components of $E$ as a canonical divisor. Its coefficients are determined by

Date: Appeared in Duke Math. Journal 159 no. 3 (2011), 539-559.

${ }^{1} 2010$ Mathematics Subject Classification. Primary 14B05; Secondary 32S25, 32S50.

Key words and phrases: Gorenstein, $\mathbb{Q}$-Gorenstein, numerically Gorenstein, normal, surface singularity, plumbing, holomorphic symplectic. 
the weighted dual graph, therefore they are topological invariants of the singularity. The coefficient of each component of $E$ is called its discrepancy. The fact that a singularity is numerically Gorenstein is a topological property: it is equivalent to the fact that, for some resolution, all discrepancies are integral.

Instead, the fact that a singularity is Gorenstein is not in general a topological property. In fact, Laufer [8, Theorem 4.3] proved that all analytical realizations of a given topology are Gorenstein if and only if one has either a Kleinian or a minimally elliptic topological type.

It seems that the problem to decide if any numerically Gorenstein normal surface singularity is homeomorphic to a Gorenstein one was left open. We learnt this problem from Jonathan Wahl (see the introduction of [15]). We prove (Theorem 4.11):

Any numerically Gorenstein normal surface singularity is homeomorphic to a Gorenstein one, having, moreover, an exceptional set of the minimal good resolution analytically isomorphic to that associated to the initial singularity.

One gets automatically a characterization of the weighted graphs which are realized by Gorenstein surface singularities: they are precisely the negative definite ones with integral discrepancies. This fact was known before in the case of weighted homogeneous surface singularities, as a consequence of Watanabe's criterion of [21, (2.9)] for such a singularity to be Gorenstein (another proof of it is given by Wahl [19, (4.4.2)]).

Our proof starts from the fact that the holomorphic cotangent bundle of a complex manifold is naturally endowed with a holomorphic symplectic form. We get in this way a holomorphic symplectic form on the canonical bundle $K_{C}=T^{*} C$ of any smooth, complex, compact curve $C$. We pull-back this form using meromorphic maps to the total spaces of convenient line bundles. In order to take care of the components of $E$ with discrepancy -1 , which are to be treated separately, we construct also special meromorphic 2 -forms on the total spaces of line bundles on $\mathbb{P}^{1}$.

The fundamental facts which make our constructions sufficient are the following:

- They lead to meromorphic 2-forms having local normal forms without moduli in holomorphic coordinates (namely, of the type $u^{a} v^{b} d u \wedge d v$ ), which allows to plumb them holomorphically.

- If the initial numerically Gorenstein singularity is not Kleinian, cusp or simple elliptic (cases when it is automatically Gorenstein), then all the components with discrepancy -1 are rational and no two of them intersect.

By plumbing tubular neighborhoods of the 0-sections using the identifications given by the coordinate systems of the normal forms, we get smooth, complex analytic surfaces endowed with a meromorphic 2-form which is symplectic outside the compact divisor obtained as the image of the 0 -sections of the line bundles. Therefore, whenever the divisor is contractible, one gets a normal Gorenstein singularity.

Teissier and Wahl suggested we extend the theorem to all normal surface singularities, the role of Gorenstein singularities being played now by the $\mathbb{Q}$-Gorenstein ones (that is, those normal singularities such that some power of the canonical bundle is holomorphically trivial in a pointed neighborhood of the singular point).

We were able to prove this extension after Veys mentioned a result of his (see Theorem 2.6), implying that if the initial normal surface singularity is not log canonical, then for 
the minimal good resolution all components of $E$ with discrepancy -1 are rational, they intersect at most two other components of E, and they are pairwise disjoint.

In order to state the extension, we need a little more vocabulary. The index of a $\mathbb{Q}$-Gorenstein singularity is the smallest exponent $r$ such that $K_{X \backslash 0}^{r}$ is holomorphically trivial and the topological index is defined in the same way for all normal singularities, by asking $K_{X \backslash 0}^{r}$ to be topologically trivial. By using the same kind of constructions as before, we prove (Theorem 5.4):

Any normal surface singularity is homeomorphic to a $\mathbb{Q}$-Gorenstein one whose index is equal to the topological index of the initial one, having, moreover, an exceptional set of the minimal good resolution analytically isomorphic to that associated to the initial singularity.

In this enlarged context, the analog of the natural holomorphic symplectic form on $K_{C}$ is a natural meromorphic 2-form of weight $r$ on the total space of $K_{C}^{r}$, for all $r \in \mathbb{N}^{*}$. Namely, in natural local coordinates it is written $p^{1-r}(d p \wedge d q)^{r}$ (see Lemma 5.1).

Wahl mentioned to us that he can prove the previous theorem in the weighted homogeneous case by a generalization of Watanabe's arguments of [21] (unpublished).

We describe now briefly the content of the paper. In Section 2 we recall the fundamental notions about normal surface singularities used in the rest of the paper. In Section 3 we construct special meromorphic 2-forms on total spaces of line bundles on smooth, compact, complex curves. In Section 4 we plumb such total spaces along weighted dual graphs, in a way which leads to the proof of Theorem 4.1. Finally, in Section 5, we prove Theorem 5.4 by generalizing the steps of the proof of Theorem 4.1 ,

\section{BACKGROUND}

In the sequel, the singularities we consider will be germs of normal complex analytic surfaces. If $(X, 0)$ denotes a singularity, tacitly $X$ will also denote a Milnor representative of it.

The divisor of a meromorphic 2 -form $\alpha$ on a smooth surface will be denoted $\operatorname{div}(\alpha)$. Outside it, such a form is holomorphic symplectic, that is, non-degenerate (as a consequence, by Darboux' theorem, it may be written $d u \wedge d v$ in convenient local coordinates in the neighborhood of any point, a fact we will not use in this paper).

A resolution of a singularity is said to be good if its exceptional divisor has normal crossings and its components are smooth. There exists a minimal good resolution, unique up to unique isomorphism.

Definition 2.1. Let $(X, 0)$ be a normal surface singularity. It is called Gorenstein if its canonical bundle is holomorphically trivial in a punctured neighborhood of 0 . It is called numerically Gorenstein if one has instead only topological triviality.

In other words, a Gorenstein singularity is one which may be endowed with a 2-form which is holomorphic and non-vanishing in a punctured neighborhood of 0 , that is, which is holomorphic symplectic there.

Starting from a normal surface singularity, denote by $(\tilde{X}, E) \stackrel{\pi}{\rightarrow}(X, 0)$ a good resolution, where $E$ is the reduced exceptional divisor. By taking pull-backs by the morphism $\pi$, one 
identifies the space of holomorphic 2-forms on $X \backslash 0$ with the one of holomorphic 2-forms on $\tilde{X} \backslash E$. In particular, one may construct a holomorphic symplectic form on $X \backslash 0$ by constructing a holomorphic symplectic form on $\tilde{X} \backslash E$. It is the approach taken in this paper.

Denote by $\Gamma$ the dual (intersection) graph of $E$ : its vertices correspond bijectively to the irreducible components of $E$ and between two distinct vertices $i$ and $j$ there are as many (unoriented) edges as the intersection number $e_{i j}:=E_{i} \cdot E_{j} \geq 0$ of the corresponding components. In particular, $\Gamma$ has no loops. Moreover, each vertex $i$ of $\Gamma$ is weighted by the self-intersection number $-e_{i}:=E_{i}^{2}<0$ of the associated component $E_{i}$ inside $\tilde{X}$, and by the genus $g_{i}$ of $E_{i}$.

Denote by $V(\Gamma)$ the set of vertices of $\Gamma$. If $i \in V(\Gamma)$, its valency is the number of edges connecting it to other vertices.

By classical results of Du Val [17] and Mumford [12], the intersection form of the weighted dual graph is negative definite. Conversely, Grauert [5] proved that if the form associated to a reduced compact divisor $E$ on a smooth surface is negative definite, then $E$ can be contracted to a normal singular point of an analytic surface, well-defined up to unique analytic isomorphisms.

As the intersection form is negative definite, there exists a unique divisor with rational coefficients $Z_{\text {can }}$ supported by $E$ such that $Z_{c a n} \cdot E_{i}=K_{\tilde{X}} \cdot E_{i}$, for all $i \in V(\Gamma)$.

Definition 2.2. The divisor $Z_{\text {can }}$ is called the canonical cycle of $E$ (or of the resolution $\pi)$. If $Z_{c a n}=\sum_{i \in V(\Gamma)} k_{i} E_{i}$, then the coefficient $k_{i}$ is called the discrepancy of $E_{i}$.

The adjunction relations, applied to the curves $E_{i}$ inside the surface $\tilde{X}$, may be written as:

$$
\left(k_{i}+1\right) e_{i}=-2 g_{i}+2+\sum_{\substack{j \in V(\Gamma) \\ j \neq i}} k_{j} e_{i j}, \forall i \in V(\Gamma) .
$$

Durfee [3] proved the following characterization of numerically Gorenstein singularities (which explains the attribute "numerically"):

Proposition 2.3. The normal surface singularity $(X, 0)$ is numerically Gorenstein if and only if for any (or some) good resolution, all discrepancies are integral.

One has the following generalization of the notion of normal Gorenstein singularity:

Definition 2.4. The normal surface singularity $(X, 0)$ is called $\mathbb{Q}$-Gorenstein if there exists a positive integer $r$ such that the pluricanonical bundle $K_{X}^{r}$ is holomorphically trivial in a punctured neighborhood of 0 . The smallest such exponent $r$ is then called the index of the singularity.

Equivalently, $(X, 0)$ is $\mathbb{Q}$-Gorenstein if and only if the canonical class $K_{X}$ (consisting a priori of Weil divisors) is (holomorphically) $\mathbb{Q}$-Cartier and the index is the smallest integral factor making it Cartier. The normal Gorenstein singularities are precisely the $\mathbb{Q}$-Gorenstein ones of index 1.

In dimension 2 , being $\mathbb{Q}$-Gorenstein is equivalent to being a cyclic quotient of a Gorenstein singularity (the canonical cover), a fact which is no longer true in higher dimensions, 
where the canonical cover may not be Cohen-Macaulay. But in this paper we don't use this view-point on $\mathbb{Q}$-Gorenstein surface singularities.

If $(X, 0)$ is $\mathbb{Q}$-Gorenstein and $K_{X}^{r}$ is holomorphically trivial on $X \backslash 0$, the pull-back by the resolution morphism $\pi$ of a holomorphic trivializing section of $K_{X}^{r}$ becomes a meromorphic section of $K_{\tilde{X}}^{r}$ whose divisor is exactly $r Z_{c a n}$. Therefore, in this case $r$ is a common denominator of the discrepancies. By the same method as the one used by Durfee to prove Proposition 2.3, one shows that the smallest common denominator of the discrepancies is the smallest exponent $r \in \mathbb{N}^{*}$ such that $K_{X}^{r}$ is topologically trivial on $X \backslash 0$. This motivates us to introduce the following:

Definition 2.5. The topological index of the singularity $(X, 0)$ is the smallest positive integer $r$ such that $K_{X \backslash 0}^{r}$ is topologically trivial.

Recall also the following special classes of singularities:

- the Kleinian singularities, also known as Du Val singularities, rational double points or simple surface singularities (see Durfee [4]) are, up to isomorphism, the surface singularities of the form $\mathbb{C}^{2} / G$, where $G$ is a finite subgroup of $S U(2)$. For these singularities, the minimal resolution is good, it has $Z_{K}=0$ and its dual graph $\Gamma$ is one of the trees $A_{n}, D_{n}, E_{6}, E_{7}, E_{8}$ with $g_{i}=0, e_{i}=2, \forall i \in V(\Gamma)$.

- the cusp singularities are those normal surface singularities which have a good resolution whose dual graph $\Gamma$ is homeomorphic to a circle and $g_{i}=0, \forall i \in V(\Gamma)$ (see Looijenga [10, page 16]).

- the simple elliptic singularities are those normal surface singularities whose minimal resolution has a smooth exceptional divisor of genus 1. As well as the cusp singularities, they are minimally elliptic (see Laufer [8]). As we do not need this last notion in the paper, we do not give more details about it.

- the log canonical singularities are those normal surface singularities whose canonical cycle satisfies the inequalities $k_{i} \geq-1, \forall i \in V(\Gamma)$. By a result of Sakai [16], they are necessarily $\mathbb{Q}$-Gorenstein, with an index equal to their topological index. One may find their classification in Sakai [16], Kawamata [6], Wahl [20], Alexeev [1] or Matsuki [11]. By this classification, one sees that the numerically Gorenstein log canonical singularities (therefore the Gorenstein ones) are exactly the Kleinian, the cusp and the simple elliptic singularities. This class may also be defined in higher dimensions. Then, one usually asks the singularity to be $\mathbb{Q}$-Gorenstein, in order to be able to define the discrepancies (an exception is the recent paper [2], in which discrepancies are defined also for singularities which are not $\mathbb{Q}$-Gorenstein). Sakai's result quoted before shows that this supplementary condition is not needed in dimension 2 (see also [11, Remark 4-6-3 (ii)]).

In [18, 2.10], Veys proved the following theorem about the behaviour of the coefficients of the canonical cycle on the dual graph of the minimal good resolution:

Theorem 2.6. Let $(X, 0)$ a normal surface singularity which is not log canonical. Denote by $V^{-}(\Gamma)$ the set of vertices $i$ of the minimal good resolution such that $k_{i}<-1$, which is non-empty by hypothesis. Then:

(1) The full subgraph $\Gamma^{-}$of $\Gamma$ with vertex set $V^{-}(\Gamma)$ is connected. 
(2) The full subgraph of $\Gamma$ with vertex-set $V(\Gamma) \backslash V^{-}(\Gamma)$ is a disjoint union of segments whose vertices represent rational components of the exceptional divisor. Moreover, exactly one end of each segment is attached (by one edge) to $\Gamma^{-}$, and the discrepancies are strictly increasing along the segment, starting from that end.

(3) All the discrepancies are (strictly) negative.

In [18, 2.11], Veys states that the previous theorem may be obtained as a corollary of results of Wahl [20]. Instead, he uses mainly results of Alexeev [1]. Before, Laufer [9. Prop. 2.1] had proved the analog of (2.6.3) for the minimal (not necessarily good) resolution of any normal surface singularity which is not Kleinian.

Remark 2.7. Veys' terminology is slightly different from ours. For instance, he uses the name $\log$ resolution instead of good resolution and he works with the $\log$ discrepancies $a_{i}:=$ $k_{i}+1$ instead of the discrepancies $k_{i}$. We prefer to work with the discrepancies because in our computations they appear explicitely as exponents of coefficients of meromorphic 2 -forms (see the last two sections).

As a corollary of (2.612), we have the following special case concerning the numerically Gorenstein singularities which are not log canonical:

Corollary 2.8. Start from a numerically Gorenstein singularity which is not smooth, Kleinian, a cusp singularity or a simple elliptic one. Then the dual graph $\Gamma$ of the minimal good resolution satisfies $k_{i} \leq-1$ for all $i \in V(\Gamma)$. Moreover, if $k_{i}=-1$, then $E_{i}$ is a smooth rational curve, $i$ is of valency 1 and the unique vertex $j$ to which it is connected satisfies $k_{j}=-2$.

Proof. The only statement which is not a special case of the previous theorem is the last one. But it is an immediate consequence of the adjunction formulae (2.1).

In fact the corollary could be proved by showing first that Laufer's theorem [9, Prop. 2.1] stated before, namely, the fact that (2.6.3) holds for the minimal resolution of a normal surface singularity which is not Kleinian, is also true for the minimal good resolution of a numerically Gorenstein singularity. This is what we did before knowing Veys' results. We analyzed what happens at each step of the sequence of blowing-ups which passes from the minimal resolution to the minimal good one and we finished by using the adjunction relations.

One has a generalization for all normal surface singularities which are not log canonical:

Corollary 2.9. Start from a normal surface singularity which is not log canonical. On the dual graph of the minimal good resolution, if $i$ is a vertex of $\Gamma$ such that $k_{i}=-1$, then it represents a smooth rational curve and its valency is 1 or 2. Moreover:

- If it is of valency 1 , then the unique vertex $j$ to which it is connected satisfies $k_{j}=-2$.

- If it is of valency 2 , then the two vertices $j_{1}, j_{2}$ to which it is connected satisfy $k_{j_{1}}+k_{j_{2}}=$ -2 , with $k_{j_{1}} \neq-1, k_{j_{2}} \neq-1$.

Proof. This is again a direct consequence of Theorem 2.6 and of the adjunction formulae.

Remark 2.10. In particular, the exceptional divisor of the minimal good resolution of non log canonical normal surface singularities does not contain adjacent components $E_{i}$ 
and $E_{j}$ such that $k_{i}=k_{j}=-1$ (see also Remark 4.2). As Veys told us, this conjectured statement was his motivation for proving Theorem 2.6.

\section{Construction of SPeCial Meromorphic 2-Forms on Line BundLeS}

Our idea for proving the theorem stated in the title of the paper, is to construct first meromorphic 2-forms on total spaces of line bundles which are holomorphic symplectic outside the union of the 0-section and of some fibers, and then to plumb them in such a way that the symplectic forms glue. This section is dedicated to the first step and the next one to the second step.

When we say that $L$ is a line bundle, $L$ will denote the total space (and not the associated sheaf of regular sections). In particular, by a form defined on $L$ we will mean a form defined on the total space of $L$.

Proposition 3.1. Let $C$ be a compact, connected and smooth complex curve of genus $g \geq 0$. Fix an integral divisor $D$ on $C$ and an integer $m \in \mathbb{Z}^{*}$ which divides $2 g-2-\operatorname{deg}(D)$. Then there exists a line bundle $L \stackrel{\psi}{\longrightarrow} C$ of degree $m^{-1}(2 g-2-\operatorname{deg}(D))$, endowed with a meromorphic 2 -form $\alpha$ such that:

(1) $\operatorname{div}(\alpha)=(m-1) C+\psi^{*}(D)$, where $C$ is seen here as the 0 -section of $L$.

(2) For each point $A \in C$, if $a \in \mathbb{Z}$ denotes the multiplicity of $D$ at that point, one has a system of local coordinates $(q, f)$ centered at $A$, seen as a point of the 0 -section of $L$, such that $q$ is constant on the fibers of $\pi$, that $f=0$ defines $C$ and moreover:

$$
\alpha=f^{m-1} q^{a} d f \wedge d q .
$$

Proof. Denote by $L_{D}$ the line bundle over $C$ whose sheaf of sections is $\mathcal{O}(D)$ and by $s_{D}$ its canonical meromorphic section defining $D$. Tensoring by $s_{D}$ one gets a meromorphic map between total spaces of line bundles:

$$
H_{D}:=K_{C} \otimes L_{-D} \cdots \stackrel{\tau_{D}}{\longrightarrow} K_{C},
$$

where $K_{C} \stackrel{\gamma}{\longrightarrow} C$ denotes the canonical line bundle of $C$, equal to its holomorphic cotangent bundle. Denote by $\alpha_{C}$ the canonical holomorphic symplectic form on the surface $K_{C}$. If $q$ denotes a local coordinate on the open set $U \subset C$ and $p$ is the associated dual coordinate on $\gamma^{-1}(U) \subset K_{C}$ (that is, the general 1-form on $U$ is written $p d q$ ), one has the following expression for $\alpha_{C}$ on $\gamma^{-1}(U)$ :

$$
\alpha_{C}=d p \wedge d q .
$$

Denote then by $\alpha_{D}$ the meromorphic 2-form on $H_{D}$ obtained as the pull-back of $\alpha_{C}$ by $\tau_{D}:$

$$
\alpha_{D}:=\tau_{D}^{*}\left(\alpha_{C}\right) .
$$

Consider now any $m$-th root $H_{D}^{1 / m}$ of the line bundle $H_{D}$ and the $m$-th tensor power meromorphic map:

$$
H_{D}^{1 / m} \stackrel{\mu_{D, m}}{\longrightarrow} H_{D}
$$


Such line bundles $H_{D}^{1 / m}$ are the solutions $H$ of the equation $m \cdot H=H_{D}$ in the Picard group $(\operatorname{Pic}(C),+)$ written additively. The set of solutions is non-empty by the hypothesis that $m \neq 0$ divides $\operatorname{deg}\left(H_{D}\right)=2 g-2-\operatorname{deg}(D)$ and it is a principal space (a torsor) under the subgroup of $|m|$-torsion elements of the Jacobian $\operatorname{Pic}^{0}(C)$ of $C$. This Jacobian is a real $(2 g)$-dimensional torus, therefore there are $|m|^{2 g}$ such line bundles. We may take any one of them.

Denote by $\alpha_{D, m}$ the meromorphic 2-form on $H_{D}^{1 / m}$ obtained as the pull-back of $\alpha_{D}$ by $\mu_{D, m}$ :

$$
\alpha_{D, m}:=\mu_{D, m}^{*}\left(\alpha_{D}\right) .
$$

We claim that the pair $(L, \alpha):=\left(H_{D}^{1 / m}, \alpha_{D, m}\right)$ satisfies both properties 1. and 2 ,

The rest of the proof is devoted to an explanation of this claim.

The map $\tau_{D} \circ \mu_{D, m}$ (see (3.1) and (3.4)) is regular and without critical points on the complement $H_{D}^{1 / m} \backslash\left(C \cup \psi_{D, m}^{-1}(|D|)\right)$, where $H_{D}^{1 / m} \stackrel{\psi_{D, m}}{\longrightarrow} C$ denotes the bundle projection and $|D|$ denotes the support of $D$. It is therefore enough to check point Q . in order to deduce also point 1 .

We will proceed in two steps, by computing first the form $\alpha_{D}$ in adapted local coordinates and then the form $\alpha_{D, m}$.

- Consider any local coordinate $q$ on $C$ centered at $A$. Choose then a local trivializing section $\sigma_{A}$ of $L_{D}$ in the neighborhood of $A$ such that $s_{D}=q^{a} \sigma_{A}$. Denote by $\left(\sigma_{A}\right)^{*}$ the dual trivializing section of $L_{-D}=L_{D}^{*}$. Then $d q \otimes\left(\sigma_{A}\right)^{*}$ is a local trivializing section of $H_{D}$, with respect to which a general section is written $h d q \otimes\left(\sigma_{A}\right)^{*}$. Then $(q, h)$ is a system of local coordinates on the total space $H_{D}$ in the neighborhood of $A$.

We have $\tau_{D}\left(h d q \otimes\left(\sigma_{A}\right)^{*}\right)=h d q s_{D}\left(\left(\sigma_{A}\right)^{*}\right)=h q^{a} d q$, which shows that with respect to the local coordinate systems $(q, h)$ on $H_{D}$ and $(q, p)$ on $K_{C}$, the map $\tau_{D}$ is given by: $(q, h) \rightarrow\left(q, h q^{a}\right)$. Combined with formula (3.2), this implies that the meromorphic 2-form $\alpha_{D}$ defined by (3.3) has the following expression in the coordinate system $(q, h)$ on $H_{D}$ :

$$
\alpha_{D}=q^{a} d h \wedge d q .
$$

- We do now the same kind of reasoning for the map $\mu_{D, m}$ (see (3.4)). Choose a local trivializing section $\eta_{A}$ of $H_{D}^{1 / m}$ in the neighborhood of $A$, such that $\mu_{D, m}\left(\eta_{A}\right)=\eta_{A}^{\otimes m}=$ $d q \otimes\left(\sigma_{A}\right)^{*}$. If a general section of $H_{D}^{1 / m}$ is written $l \eta_{A}$, then $(q, l)$ is a local coordinate system on the total space $H_{D}^{1 / m}$ in the neighborhood of the point $A$. With respect to the local coordinate systems $(q, l)$ on $H_{D}^{1 / m}$ and $(q, h)$ on $H_{D}$, the map $\mu_{D, m}$ is given by $(q, l) \rightarrow\left(q, l^{m}\right)$. Combined with formula (3.6), this implies that the meromorphic 2 -form $\alpha_{D, m}$ defined by (3.5) has the following expression in the coordinate system $(q, l)$ on $H_{D}^{1 / m}$ :

$$
\alpha_{D, m}=m q^{a} l^{m-1} d l \wedge d q .
$$

By making the change of variables $f:=m^{1 / m} l$, where $m^{1 / m}$ is an arbitrary $m$-th root of $m$ (which exists, by the hypothesis $m \neq 0$ ), we get from equation (3.7):

$$
\alpha_{D, m}=f^{m-1} q^{a} d f \wedge d q .
$$

The proposition is proved. 
Remark 3.2. The statement (3.12) is not a consequence of the statement (3.1]1.). Indeed, there are moduli for germs of meromorphic 2-forms whose divisor has normal crossings with two irreducible components, implying that not all germs of meromorphic 2 -forms whose divisor has normal crossings may be written in the form $u^{a} v^{b} d u \wedge d v$ in adapted local coordinates. One gets the simple normal forms stated in (3.1.2.) as a consequence of our construction by successive pull-backs of the standard symplectic form on the canonical bundle.

The previous proposition does not allow to get meromorphic 2-forms having a pole of order 1 along the 0 -section. In the next one, we construct special meromorphic 2 -forms on the total spaces of arbitrary line bundles on a smooth rational curve, having such pole of order 1 along the 0 -section. We will see that this is enough to prove the characterization of the topological types of Gorenstein singularities.

Proposition 3.3. Let $C$ be a compact, connected and smooth rational curve. Let $L \stackrel{\psi}{\longrightarrow} C$ be a line bundle on it and $A$ be a point on $C$. Then there exists a meromorphic 2-form $\alpha$ on $L$ such that:

(1) $\operatorname{div}(\alpha)=-C-2 \psi^{*}(A)$;

(2) one has a system of local coordinates $(q, f)$ centered at $A$, seen as a point of the 0 -section of $L$, such that $q$ is constant on the fibers of $\psi$, that $f=0$ defines $C$ and moreover:

$$
\alpha=f^{-1} q^{-2} d f \wedge d q .
$$

Proof. Choose a covering of $C$ by two affine charts isomorphic to $\mathbb{C}$, one with coordinate $q_{1}$ centered at $A$ and the other with coordinate $q_{2}$, such that:

$$
q_{2}=q_{1}^{-1} \text {. }
$$

Choose then a trivialization of $L$ over the $q_{1}$-chart, with respect to which the fiber coordinate is denoted $f_{1}$ and another trivialization over the $q_{2}$-chart, with respect to which the fiber-coordinate is denoted $f_{2}$, such that the change of trivialization over the overlap is given by the equation:

$$
f_{2}=q_{1}^{n} f_{1} .
$$

Here $n=\operatorname{deg}(L)$. Such trivializations exist because on $\mathbb{P}^{1}$ any line bundle of degree $n$ is isomorphic to $\mathcal{O}(n)$. Therefore:

$$
d f_{2} \wedge d q_{2}=-q_{1}^{n-2} d f_{1} \wedge d q_{1} .
$$

Using also the equations (3.8) and (3.9), this implies that the meromorphic 2-form which on the chart with coordinates $\left(q_{2}, f_{2}\right)$ of $L$ is given by $\alpha:=-f_{2}^{-1} d f_{2} \wedge d q_{2}$, becomes $\alpha=f_{1}^{-1} q_{1}^{-2} d f_{1} \wedge d q_{1}$ in the chart with coordinates $\left(q_{1}, f_{1}\right)$. Looking at the expressions of $\alpha$ in both charts, we see that the proposition is proved by taking $(q, f):=\left(q_{1}, f_{1}\right)$.

\section{The topology of Gorenstein normal surface Singularities}

We are ready to establish the theorem announced in the title of the paper. The idea is to plumb using biholomorphic maps the line bundles constructed using Proposition 3.1 and Proposition 3.3 , in such a way that the associated meromorphic 2-forms glue. 
We recall that the operation of plumbing of tubular neighborhoods of 0 -sections of line bundles over compact Riemann surfaces was introduced by Mumford in [12. Since then, this operation was nevertheless mainly used in the differentiable category, that is, without necessarily respecting holomorphic structures. Instead, in our approach it is essential to do holomorphic plumbing. This procedure was also used by Laufer (see e.g. [7, page 83]).

Theorem 4.1. Consider a numerically Gorenstein surface singularity. Then it is homeomorphic to a normal Gorenstein surface singularity. Moreover, the second singularity may be chosen such that the exceptional curve of its minimal good resolution is analytically isomorphic to that of the initial singularity.

Proof. Let $(X, 0)$ be a numerically Gorenstein singularity and $(\tilde{X}, E) \stackrel{\pi}{\longrightarrow}(X, 0)$ its minimal good resolution. If the singularity is smooth, Kleinian, a cusp singularity or a simple elliptic one, then it is automatically Gorenstein (see Laufer [8]). Therefore, we may suppose that we are not in one of these cases. Consequently, we may apply Corollary 2.8,

For each vertex $i$ of $\Gamma$, denote by $V_{i} \subset V(\Gamma)$ the set of indices of those components of $E$ which have non-empty intersection with $E_{i}$. For every $j \in V_{i}$, denote $E_{i, j}:=E_{i} \cap E_{j}$. This set may consist of several points: $E_{i, j}=\left\{A_{i, j, l} \mid l \in I(i, j)\right\}$. Of course, $E_{i, j}=E_{j, i}$, which allows us to choose the index sets such that $A_{i, j, l}=A_{j, i, l}$ for all $l \in I(i, j)=I(j, i)$. We will look at $E_{i, j}$ also as a reduced divisor on the abstract curve $E_{i}$.

By Corollary 2.8, we have $k_{i} \leq-1$ for all $i \in I$. We consider two cases:

- Suppose that $k_{i}<-1$. Then apply Proposition 3.1 to $C:=E_{i}, g:=g_{i}, m:=k_{i}+1$, $D:=\sum_{j \in V_{i}} k_{j} E_{i, j}$. The hypothesis that $m$ divides $2 g-2-\operatorname{deg}(D)$ is a consequence of formula (2.1).

- Suppose that $k_{i}=-1$. By Corollary 2.8, we know that $E_{i}$ is then a smooth rational curve and that $V_{i}$ has only one element $j$, which satisfies moreover $k_{j}=-2$. Then apply Proposition 3.3 to $C:=E_{i}, L:=\mathcal{O}\left(-e_{i}\right),\{A\}=E_{i} \cap E_{j}$.

By combining both cases, we see that for all $i \in V(\Gamma)$, there exists a line bundle $L_{i} \stackrel{\psi_{i}}{\longrightarrow} E_{i}$ of degree $-e_{i}$ on $E_{i}$ and a meromorphic 2-form $\alpha_{i}$ on it, such that:

- $\operatorname{div}\left(\alpha_{i}\right)=k_{i} E_{i}+\sum_{j \in V_{i}} k_{j} \psi_{i}^{*}\left(E_{i, j}\right)$;

- for each point $A_{i, j, l} \in E_{i, j}$, one has a system of local holomorphic coordinates $\left(q_{i, j, l}, f_{i, j, l}\right)$ centered at $A_{i, j, l}$ on the total space $L_{i}$, such that $q_{i, j, l}$ is constant on the fibers of $\psi_{i}$, that $f_{i, j, l}=0$ defines the 0 -section $E_{i} \subset L_{i}$ and moreover:

$$
\alpha_{i}=\left(q_{i, j, l}\right)^{k_{j}}\left(f_{i, j, l}\right)^{k_{i}} d f_{i, j, l} \wedge d q_{i, j, l} .
$$

Consider then neighborhoods of the 0-sections $E_{i} \subset L_{i}$, and plumb them by using the following holomorphic identifications of neighborhoods of the points $A_{i, j, l}$ and $A_{i, j, l}$ contained in the previous charts, whenever $i$ and $j$ are adjacent vertices of $\Gamma$ and $l \in I(i, j)$ :

$$
\left\{\begin{array}{l}
q_{i, j, l}=\lambda_{i, j} f_{j, i, l} \\
q_{j, i, l}=\lambda_{j, i} f_{i, j, l}
\end{array} \quad \text { with } \lambda_{i, j}, \lambda_{j, i} \in \mathbb{C}^{*} .\right.
$$

The non-vanishing complex numbers $\lambda_{i, j}, \lambda_{j, i}$ are to be chosen such that by this change of coordinates, the form $\alpha_{i}$ given by (4.1) is transformed into $\alpha_{j}$, which says precisely that they glue on the surface obtained by the plumbing. 
These compatibility conditions translate into the following system of equations, which is non-trivial because, when we plumb, we permute the order of the coordinates:

$$
\lambda_{i, j}^{k_{j}+1}=-\lambda_{j, i}^{k_{i}+1} \text { for all pairs }(i, j) \text { of adjacent vertices of the graph } \Gamma .
$$

As before, our hypothesis allows us to apply Corollary 2.8, which implies that there is no pair of adjacent vertices such that $k_{i}=k_{j}=-1$ (otherwise, equation (4.2) would be contradictory). In fact this is the only obstruction to the existence of solutions of the system (4.2): orient each edge of $\Gamma$ such that, whenever an edge goes from a vertex $i$ to a vertex $j$, we have $k_{j}<-1$. Define then $\lambda_{j, i}:=1$ and $\lambda_{i, j}$ to be a $\left(k_{j}+1\right)$-th root of -1 . We get obviously a solution of (4.2).

After such a choice, the surface $\tilde{Y}$ obtained by the plumbing is smooth complex analytic and the images of the 0-sections $E_{i} \subset L_{i}$ form a divisor canonically identified to $E$ and with the given weighted dual graph. By Grauert's theorem (see [5]), this divisor $E$ may be contracted in $\tilde{Y}$, yielding a normal singularity $(Y, 0)$. By our construction, the meromorphic 2-forms $\alpha_{i}$ glue into a meromorphic 2-form $\tilde{\alpha}$ on $\tilde{Y}$, which is symplectic outside $E$. Therefore, it descends into a holomorphic symplectic form on $Y \backslash 0$, which means that $(Y, 0)$ is Gorenstein.

The second statement of the theorem is a consequence of the fact that, in our construction, one starts only from an abstract compact curve $E=\cup_{i \in V(\Gamma)} E_{i}$ with simple normal crossings, decorated with weights $e_{i}$ such that the associated intersection form is negative definite and such that the coefficients $k_{i}$ determined by the adjunction system (2.1) are integers. As a result of our construction, one gets a Gorenstein singularity having a resolution whose exceptional divisor is $E$, and such that $E_{i}^{2}=-e_{i}$ for each irreducible component $E_{i}$.

The theorem is proved.

Remark 4.2. We could do the plumbing construction using only the meromorphic 2forms described in Propositions 3.1 and 3.3, because we knew that we could work with a good resolution such that for any two intersecting components $E_{i}, E_{j}$ of the exceptional divisor, one does not have simultaneously $k_{i}=k_{j}=-1$ (see also Remark 2.10). Indeed, in Proposition 3.1 one has $m-1 \neq-1$ and in Proposition 3.3 one has adjacent components of the divisor of the meromorphic 2-form with orders 1 and 2 (compare to Corollary 2.8).

Remark 4.3. Our construction yields singularities whose minimal good resolution has the following special property: each irreducible component of the exceptional divisor has a neighborhood isomorphic to a neighborhood of the 0-section of its normal bundle.

The following corollary settles a question left open by Neumann and Wahl [14]:

Corollary 4.4. Any normal surface singularity whose boundary is an integral homology sphere is homeomorphic to a Gorenstein normal surface singularity.

Proof. The boundary is an integral homology sphere if and only if $\Gamma$ is a tree with $g_{i}=0$ for all $i \in V(\Gamma)$ and the determinant of the intersection form is \pm 1 . Therefore, the adjunction system (2.1) shows that, in this case, the discrepancies are all integral. We conclude using Theorem 4.1. 


\section{Extension to $\mathbb{Q}$-Gorenstein Singularities}

If $S$ is a smooth complex manifold of dimension $s>0$ and $r \in \mathbb{Z}^{*}$, we will say that the holomorphic (meromorphic) sections of the pluricanonical line bundle $K_{S}^{r}$ of exponent $r$ are holomorphic (meromorphic) s-forms of weight $r$. If $\left(q_{1}, \ldots, q_{s}\right)$ is a system of local coordinates on $S$, such a form is written canonically as :

$$
f\left(q_{1}, \ldots, q_{s}\right)\left(d q_{1} \wedge \cdots \wedge d q_{s}\right)^{r}
$$

where $f$ is holomorphic (meromorphic).

In particular, consider $S=C$, a smooth holomorphic curve (a Riemann surface). Denote by: $K_{C}^{r} \stackrel{\gamma_{r}}{\longrightarrow} C$ the projection morphism. If $q$ is a local coordinate on the open set $U \subset C$, then $(d q)^{r}$ is a local section of $\left.K_{C}^{r}\right|_{U}$. One may take as a second coordinate on $\gamma_{r}^{-1}(U)$ the analog of the dual coordinate $p$ of the case $r=1$ (see the beginning of the proof of Proposition 3.1), that is, the holomorphic function $p_{r}$ such that the sections $\alpha$ of $\left.K_{C}^{r}\right|_{U}$ (that is, the 1-forms of weight $r$ ) are written as:

$$
\alpha=p_{r}(d q)^{r} .
$$

The next lemma shows that all pluricanonical bundles over a curve are endowed with natural meromorphic 2-forms of the same weight as the exponent of the bundle, generalizing the existence of the natural symplectic form on the canonical bundle.

Lemma 5.1. Let $C$ be a smooth holomorphic curve and $r \in \mathbb{Z}^{*}$. Then the holomorphic 2-forms of weight $r$ on the total space $K_{C}^{r}$ which are expressed in local coordinates as follows:

$$
p_{r}^{1-r}\left(d p_{r} \wedge d q\right)^{r}
$$

glue into a well-defined meromorphic 2-form $\alpha_{C, r}$ of weight $r$ on the total space $K_{C}^{r}$.

Proof. Let $\tilde{q}$ be a second local coordinate on $C$, defined on an open set overlapping the domain of definition of $q$. Denote by $\tilde{p}_{r}$ the associated complementary coordinate on the total space $K_{C}^{r}$. Then $\tilde{p}_{r}(d \tilde{q})^{r}=p_{r}(d q)^{r}$, which implies:

$$
\tilde{p}_{r}=p_{r} \cdot\left(\frac{d \tilde{q}}{d q}\right)^{-r}
$$

As a consequence, $d \tilde{p}_{r} \wedge d \tilde{q}=\left(\frac{d \tilde{q}}{d q}\right)^{-r+1} d p_{r} \wedge d q$ which implies, by taking $r$-th powers on both sides and by applying again relation (5.2), the desired equality:

$$
\tilde{p}_{r}^{1-r}\left(d \tilde{p}_{r} \wedge d \tilde{q}\right)^{r}=p_{r}^{1-r}\left(d p_{r} \wedge d q\right)^{r} .
$$

The following proposition generalizes Proposition 3.1. It specializes to it for $r=1$.

Proposition 5.2. Let $C$ be a compact, connected and smooth complex curve of genus $g \geq 0$. Fix an integral divisor $D$ on $C$, an integer $r \in \mathbb{N}^{*}$ and an integer $m \in \mathbb{Z}^{*}$ which divides $r(2 g-2)-\operatorname{deg}(D)$. Then there exists a line bundle $L \stackrel{\psi}{\longrightarrow} C$ of degree $m^{-1}(r(2 g-2)-\operatorname{deg}(D))$, endowed with a meromorphic 2 -form $\alpha$ of weight $r$, such that:

(1) $\operatorname{div}(\alpha)=(m-r) C+\psi^{*}(D)$, where $C$ is seen here as the 0 -section of $L$. 
(2) For each point $A \in C$, if $a \in \mathbb{Z}$ denotes the multiplicity of $D$ at that point, one has a system of local coordinates $(q, f)$ centered at $A$, seen as a point of the 0 -section of $L$, such that $q$ is constant on the fibers of $\psi$, that $f=0$ defines $C$ and moreover:

$$
\alpha=f^{m-r} q^{a}(d f \wedge d q)^{r} .
$$

Proof. We follow the steps of the proof of Proposition 3.1. Consider the natural meromorphic map got by tensoring with the meromorphic section $s_{D}$ of $L_{D}$ :

$$
H_{D, r}:=K_{C}^{r} \otimes L_{-D} \stackrel{\tau_{D, r}}{\longrightarrow} K_{C}^{r}
$$

Then $(d q)^{r} \otimes \sigma_{A}^{*}$ is a trivializing section of $K_{C}^{r} \otimes L_{-D}$. If $h_{r}(d q)^{r} \otimes \sigma_{A}^{*}$ denotes the general section of $H_{D, r}$, then $\left(q, h_{r}\right)$ is a local coordinate system on $H_{D, r}$ in the neighborhood of $A$. With respect to the coordinate systems $\left(q, h_{r}\right)$ and $\left(q, p_{r}\right)$, the map $\tau_{D, r}$ is given by $\left(q, h_{r}\right) \rightarrow\left(q, h_{r} q^{a}\right)$. Recalling that $\alpha_{C, r}$ was defined by Lemma 5.1, this implies that:

$$
\left(\tau_{D, r}\right)^{*} \alpha_{C, r}=\left(\tau_{D, r}\right)^{*}\left(p_{r}^{1-r}\left(d p_{r} \wedge d q\right)^{r}\right)=\left(h_{r} q^{a}\right)^{1-r}\left(d\left(h_{r} q^{a}\right) \wedge d q\right)^{r}=h_{r}^{1-r} q^{a}\left(d h_{r} \wedge d q\right)^{r} .
$$

Consider now an arbitrary $m$-th root $H_{D, r}^{1 / m}$ of the line bundle $H_{D, r}$, which exists by the hypothesis that $m \neq 0$ divides $\operatorname{deg}\left(H_{D, r}\right)=r(2 g-2)-\operatorname{deg}(D)$. In adapted local coordinates, chosen as in the proof of Proposition 3.1, the $m$-th tensor power map

$$
H_{D, r}^{1 / m} \stackrel{\mu_{D, r, m}}{\longrightarrow} H_{D, r}
$$

is given by $(q, l) \rightarrow\left(q, l^{m}\right)$. Therefore:

$$
\alpha_{D, r, m}:=\left(\mu_{D, r, m}\right)^{*}\left(\tau_{D, r}\right)^{*} \alpha_{C, r}=l^{m(1-r)} q^{a}\left(m l^{m-1} d l \wedge d q\right)^{r}=m^{r} l^{m-r} q^{a}(d l \wedge d q)^{r} .
$$

By making the change of variables $f:=m^{r / m} l$, where $m^{1 / m}$ is an arbitrary $m$-th root of $m$, we get:

$$
\alpha_{D, r, m}=f^{m-r} q^{a}(d f \wedge d q)^{r} .
$$

This shows that the pair $(L, \alpha):=\left(H_{D, r}^{1 / m}, \alpha_{D, r, m}\right)$ satisfies both properties 1. and 2.

In the same way as Proposition 3.1 did not allow to get meromorphic 2-forms with poles of order 1 along the 0 -section, the previous one does not allow to get meromorphic 2 -forms of weight $r$ with poles of order $r$ along the 0 -section. We get such poles using the following proposition, which generalizes Proposition 3.3. It specializes to it for $r=1, m_{1}=-2$ and $m_{2}=0$.

Proposition 5.3. Let $C$ be a compact, connected and smooth rational curve. Let $L \stackrel{\psi}{\rightarrow} C$ be a line bundle on it and $A_{1}, A_{2}$ be two distinct points on $C$. Fix an integer $r \in \mathbb{N}^{*}$ and two other integers $m_{1}, m_{2} \in \mathbb{Z} \backslash\{-r\}$ such that $m_{1}+m_{2}=-2 r$. Then there exists a meromorphic 2-form $\alpha$ of weight $r$ on $L$ such that:

(1) $\operatorname{div}(\alpha)=-r C+m_{1} \psi^{*}\left(A_{1}\right)+m_{2} \psi^{*}\left(A_{2}\right)$.

(2) For each $l \in\{1,2\}$, one has a system of local coordinates $\left(u_{l}, v_{l}\right)$ centered at $A_{l}$, seen as a point of the 0 -section of $L$, such that $u_{l}$ is constant on the fibers of $\psi$, that $v_{l}=0$ defines $C$ and moreover:

$$
\alpha=v_{l}^{-r} u_{l}^{m_{l}}\left(d v_{l} \wedge d u_{l}\right)^{r} .
$$


Proof. We follow the notations introduced in the proof of Proposition 3.3. That is, we work inside the charts $\left(q_{1}, f_{1}\right),\left(q_{2}, f_{2}\right)$, chosen moreover such that $A_{l}$ is defined by $q_{l}=f_{l}=0$. Consider the following meromorphic 2-form of weight $r$ on $L$ :

$$
\alpha:=f_{2}^{-r} q_{2}^{m_{2}}\left(d f_{2} \wedge d q_{2}\right)^{r} .
$$

The equations (3.8), (3.9), (3.10) imply immediately that, written in the other chart:

$$
\alpha=(-1)^{r} f_{1}^{-r} q_{1}^{m_{1}}\left(d f_{1} \wedge d q_{1}\right)^{r} .
$$

We almost get the desired theorem, with the exception of the fact that in this second chart, one has the extra factor $(-1)^{r}$. We have to care about it, in order to get the same shape for all the local normal forms, adapted to the plumbing operations to be done in the sequel (in the proof of Theorem 5.4).

- If $r$ is even, there is nothing more to be done.

- If $r$ is odd, then we do the change of variable $q_{1}=\nu \cdot u_{1}$, where $\nu$ is an arbitrary $\left(m_{1}+r\right)$-th root of $(-1)^{r}$, which exists, by the hypothesis $m_{1} \neq-r$. We get: $\alpha=$ $f_{1}^{-r} u_{1}^{m_{1}}\left(d f_{1} \wedge d u_{1}\right)^{r}$. By taking $u_{2}:=q_{2}$ and $v_{l}:=f_{l}$ for $l \in\{1,2\}$, we see that $\alpha$ is expressed as in point [2. in both charts with coordinates $\left(u_{l}, v_{l}\right)$. As these charts cover $L$, the proposition is proved.

Now we are ready to prove a generalization of Theorem 4.1 to all normal surface singularities. It specializes to it for $r=1$.

Theorem 5.4. Consider a normal surface singularity, with topological index $r \geq 1$. Then it is homeomorphic to a $\mathbb{Q}$-Gorenstein singularity with index $r$. In particular, being $\mathbb{Q}$ Gorenstein imposes no topological restrictions on normal surface singularities. Moreover, the second singularity may be chosen such that the exceptional curve of its minimal good resolution is analytically isomorphic to that of the initial singularity.

Proof. The proof follows the same steps as the one of Theorem 4.1 and it uses the same notations. For each vertex $i \in \Gamma$, we consider a convenient line bundle endowed with a meromorphic 2-form of weight $r$, constructed either by Proposition 5.2, or by Proposition 5.3. We consider three cases, according to the values of the discrepancy $k_{i}$ and of the valency $k_{i}$. When $k_{i}=-1$, we know by Corollary 2.9 that the valency $v_{i}$ is either 1 or 2 and that $E_{i}$ is a rational curve.

- Suppose that $k_{i} \neq-1$. Then apply Proposition 5.2 to $C:=E_{i}, g:=g_{i}, m:=r\left(k_{i}+1\right)$, $D:=\sum_{j \in V_{i}} r \cdot k_{j} E_{i, j}$. The hypothesis that $m$ divides $r(2 g-2)-\operatorname{deg}(D)$ is a consequence of formula (2.1).

- Suppose that $k_{i}=-1$ and $v_{i}=1$. Denote by $j$ the unique element of $V_{i}$. Then apply Proposition 5.3 to $C:=E_{i}, L:=\mathcal{O}\left(-e_{i}\right),\left\{A_{1}\right\}=E_{i} \cap E_{j}, m_{1}=-2 r$ and $m_{2}=0$.

- Suppose that $k_{i}=-1$ and $v_{i}=2$. Corollary 2.9 tells us that, if $j_{1} \neq j_{2}$ are the two elements of $V_{i}$, then $k_{j_{1}}+k_{j_{2}}=-2$ and $k_{j_{1}} \neq-1, k_{j_{2}} \neq-1$. Then apply Proposition 5.3 to $C:=E_{i}, L:=\mathcal{O}\left(-e_{i}\right),\left\{A_{l}\right\}=E_{i} \cap E_{j_{l}}$ and $m_{l}=r \cdot k_{j_{l}}$, for each $l \in\{1,2\}$.

By combining the three cases, we see that for all $i \in V(\Gamma)$, there exists a line bundle $L_{i} \stackrel{\psi_{i}}{\longrightarrow} E_{i}$ of degree $-e_{i}$ on $E_{i}$ and a meromorphic 2-form $\alpha_{i}$ of weight $r$ on it, such that:

- $\operatorname{div}\left(\alpha_{i}\right)=r \cdot k_{i} E_{i}+\sum_{j \in V_{i}} r \cdot k_{j} \psi_{i}^{*}\left(E_{i, j}\right)$;

- for each point $A_{i, j, l} \in E_{i, j}$, one has a system of local holomorphic coordinates $\left(q_{i, j, l}, f_{i, j, l}\right)$ centered at $A_{i, j, l}$ on the total space $L_{i}$, such that $q_{i, j, l}$ is constant on the 
fibers of $\psi_{i}$, that $f_{i, j, l}=0$ defines the 0 -section $E_{i} \subset L_{i}$ and moreover:

$$
\alpha_{i}=\left(f_{i, j, l}\right)^{r \cdot k_{i}}\left(q_{i, j, l}\right)^{r \cdot k_{j}}\left(d f_{i, j, l} \wedge d q_{i, j, l}\right)^{r} .
$$

Then, we plumb neighborhoods of the 0-sections of these bundles by identifying neighborhoods of the points $A_{i, j, l}$ and $A_{j, i, l}$, in such a way that the forms $\alpha_{i}$ and $\alpha_{j}$ glue. Again, this is possible because we have the simple normal forms (5.3), and because no two adjacent vertices $i$ and $j$ of $\Gamma$ satisfy $k_{i}=k_{j}=-1$, a fact ascertained by the Corollary 2.9 of Veys' Theorem 2.6 (the analog of equation (4.2) is now the equation $\left.\lambda_{i, j}^{r\left(k_{j}+1\right)}=(-1)^{r} \lambda_{j, i}^{r\left(k_{i}+1\right)}\right)$. After contracting the union of the 0-sections of the bundles $L_{i}$, one gets a singularity $(X, 0)$ such that the 2-form of weight $r$ obtained by gluing the forms $\alpha_{i}$ gives a holomorphic trivialization of $K_{X \backslash 0}^{r}$. The theorem is proved.

The analog of Remark 4.3 holds obviously also in this more general context.

To conclude, we mention that the problems of characterizing the topological types of isolated complete intersections, hypersurfaces and smoothable isolated surface singularities are still open. It would also be interesting to determine whether the classes of $\mathbb{Q}$-Gorenstein singularities constructed in this paper are extremal among the $\mathbb{Q}$-Gorenstein singularities of the same topological type, for some analytical invariant.

\section{Acknowledgments}

I decided to do this research after collaborating with José Seade on [15. I am extremely grateful to him for his kind encouragements and for his remarks on a previous version of this paper. I am also grateful to Jonathan Wahl for having told me the problem and for providing several remarks on earlier versions. Moreover, I am grateful both to him and to Bernard Teissier for having asked if I could extend Theorem 4.1 to $\mathbb{Q}$-Gorenstein singularities, and to Wim Veys for having told me about his paper [18].

\section{REFERENCES}

[1] V. Alexeev. Log canonical surface singularities: arithmetical approach. In Flips and abundance for algebraic threefolds. J. Kollár ed., Astérisque 211 (1992), 47-58.

[2] S. Boucksom, T. De Fernex, C. Favre. The volume of an isolated singularity. arXiv:1011.2847.

[3] A. Durfee. The signature of smoothings of complex surface singularities. Math. Annalen 232 (1978), 85-98.

[4] A. Durfee. Fifteen characterizations of rational double points and simple critical points. Enseignement Math. 25 (1979), 131-163.

[5] H. Grauert. Über Modifikationen und exzeptionnelle analytische Mengen. Math. Annalen 146 (1962), 331-368.

[6] Y. Kawamata. Crepant blowing-ups of three dimensional canonical singularities and its application to degeneration of surfaces. Annals of Maths. 127 (1988), 93-163.

[7] H. B. Laufer. Deformations of resolutions of two-dimensional singularities. Rice Univ. Studies 59 (1) (1973), 53-96.

[8] H. B. Laufer. On minimally elliptic singularities. American Journal of Maths. 99 No. 6 (1977), 1257-1295.

[9] H. B. Laufer. The multiplicity of isolated two-dimensional hypersurface singularities. Transactions of the Amer. Math. Soc. 302, No. 2 (1987), 489-496.

[10] E. Looijenga. Isolated singular points on complete intersections. London Math. Soc. LNS 77. Cambridge University Press, 1984.

[11] K. Matsuki. Introduction to the Mori program. Springer, 2002. 
[12] D. Mumford. The topology of normal singularities of an algebraic surface and a criterion for simplicity. Publ. Math. I.H.E.S. 9 (1961), 229-246.

[13] W. Neumann. A calculus for plumbing applied to the topology of complex surface singularities and degenerating complex curves. Transactions of the Amer. Math. Soc. 268, 2 (1981), 299-344.

[14] W. Neumann, J. Wahl. Complex surface singularities with integral homology sphere links. Geom. Topol. 9 (2005), 757-811.

[15] P. Popescu-Pampu, J. Seade. A finiteness theorem for dual graphs of surface singularities. International Journ. of Maths. 20 No. 8 (2009), 1057-1068.

[16] F. Sakai. Classification of normal surfaces. Proc. of Symposia in Pure Maths. 46 (1987), 451-465.

[17] P. Du Val. On absolute and non-absolute singularities of algebraic surfaces. Revue de la Faculté des Sciences de l'Univ. d'Istanbul (A) 91 (1944), 159-215.

[18] W. Veys. Stringy invariants of normal surfaces. J. Algebraic Geometry 13 (2004), 115-141.

[19] J. Wahl. Deformations of quasi-homogeneous surface singularities. Math. Annalen 280 (1988), 105-128.

[20] J. Wahl. A characteristic number for links of surface singularities. Journal of the Amer. Math. Soc. 3 (1990), 625-637.

[21] K. Watanabe. Some remarks concerning Demazure's construction of normal graded rings. Nagoya Math. J. 83 (1981), 203-211.

Patrick Popescu-Pampu,

Université Lille 1, UFR de Mathématiques

Bâtiment M2, Cité Scientifique

59655, Villeneuve d'Ascq Cedex

France.

patrick.popescu@math.univ-lille1.fr 